Research Paper

\title{
Synaptotagmin7 Is Overexpressed In Colorectal Cancer And Regulates Colorectal Cancer Cell Proliferation
}

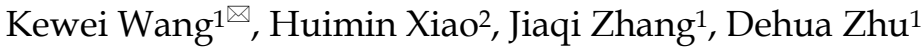 \\ 1. Department of Gastrointestinal \& hernia Surgery, First Hospital of China Medical University, Shenyang, China. \\ 2. Department of General Surgery, People's Hospital of China Medical University, Shenyang, China \\ $\triangle$ Corresponding author: Kewei Wang, Department of Gastrointestinal \& hernia Surgery, First Hospital of China Medical University, No.155 Nanjing North \\ Street, Shenyang 110001, China. Tel: +86-24-83282881; Fax: +86-24-83282886; E-mail: kwwang@cmu.edu.cn \\ (C) Ivyspring International Publisher. This is an open access article distributed under the terms of the Creative Commons Attribution (CC BY-NC) license \\ (https://creativecommons.org/licenses/by-nc/4.0/). See http://ivyspring.com/terms for full terms and conditions.
}

Received: 2018.01.23; Accepted: 2018.05.01; Published: 2018.06.12

\begin{abstract}
Purpose: Synaptotagmin7 (SYT7) belongs to the synaptotagmin gene family and plays an important role in synaptic transmission. However, the function of this gene in most human cancer especially in colorectal cancer (CRC) remains unknown. In this research, we examined SYT7's role in CRC and tried to reveal its underlying mechanism.

Methods: We examined SYT7's expression levels in normal colorectal tissue and CRC tissues from 83 patients and analyzed the possible correlation between the expression level of SYT7 and pathological characteristics. The influences of SYT7 knockdown on cell growth were detected by Celigo image cytometer, colony formation assay, cell cycle analysis and apoptosis assay in vitro. The possible molecular mechanism was assessed using a microarray and Ingenuity Pathway Analysis.

Results: Our results show that the expression of SYT7 is upregulated in colorectal cancer tissues in comparison with normal tissues and positively correlated with the pathological stage of colorectal cancer. $(P=0.015)$. We examined SYT7's role in human colorectal cancer cell line RKO by using SYT7-shRNA and revealed that SYT7 knockdown inhibit cell proliferation $(P=8.6 E-5)$, clonogenic ability $(P=4.5 E-6)$ and promoted $G 2 / M$ Phase arrest and apoptosis $(P=4.6 E-7)$. Multiple cancer-associated pathways regulated by SYT7 were unraveled by microarray and Ingenuity Pathway Analysis.

Conclusions: Our study suggests that SYT7 plays an important role in the development of CRC and SYT7 may become a new therapeutic target in CRC.
\end{abstract}

Key words: Synaptotagmin7, colorectal cancer, proliferation, apoptosis, TCGA

\section{Introduction}

Colorectal cancer (CRC) is the third most common malignancy tumor and the fourth most common cause of death by cancer in the world. The number of patients with new onset is about 12 million a year, and the deaths of patients were 600000[1]. In China, colorectal cancer is the fifth most common cancers among males, and fourth among females [2]. Radical resection is the best method for the treatment of CRC. Although chemotherapy drugs are widely used in the adjuvant treatment of CRC, drug resistance is still the main reason for the failure of cancer chemotherapy. Therefore, some new molecular markers and their roles in CRC need to be found for the better targeted therapy. With the development of plentiful open data resources, it is now potential for researchers to discover cancer-related genes more conveniently in recent years [3-5]. By using an analysis of TCGA database we found SYT7 displayed a much higher change between paired normal and cancer tissues (with a mean 3.01 fold-change in TCGA-colorectal adenocarcinoma dataset, $\mathrm{p}<0.001$ ). We thus chose SYT7 as a candidate gene for the further research. SYT7 belongs to a member of the synaptotagmin gene family, which is comprised of 17 
human isoforms [6]. STY7 encodes a protein that is similar to other synaptotagmin members mediating calcium-dependent regulation of membrane that traffics in synaptic transmission. Lysosome exocytosis and hormone secretion is mediated by a similar protein in rodents [7-11].

However, the function of this gene in human cancer especially in colorectal cancer remains unknown. In our present research, we find that SYT7 is overexpressed in CRC patients and promotes colorectal cancer cell proliferation in vitro.

\section{Material and Methods}

\section{Bioinformatics analysis}

To discover possible colorectal adenocarcinomarelated genes, we firstly analyzed the TCGA datasets. Level 3 TCGA data: TCGA_COAD_RNAseqV2 and TCGA_READ_RNAseqV2 were downloaded at the website of The Genomic Data Commons (GDC) (https:/ / portal.gdc.cancer.gov/), containing 41 and 9 paired normal colorectal tissue samples respectively. All mRNA expression values were normalized. RNA-Seq by Expectation-Maximization (RSEM) expression values were used to determine the expression levels. T-test was used to compare the two groups.

\section{Tissue Samples}

83 pairs of normal colorectal tissues and colorectal cancer tissues were gathered from patients in the First Hospital of China Medical University. All pathological diagnoses were confirmed, and were analyzed following the criterion that was depicted in the 7th edition of Union for International Cancer Control (UICC) TNM classification [12]. All normal colorectal tissues were sampled $\geq 20 \mathrm{~mm}$ away from the cancer lesions. The patients' clinical and pathological characteristics are summed up in Table 1. This research was approved by China Medical University's Ethics Committee (2017-56-2). Written informed consent was acquired from all patients whose tissue samples were used in this study.

\section{Cell line and culture}

The human colon cancer cell line RKO was acquired from the American Type Culture Collection (ATCC, Manassas, VA, USA). RKO cells were grown in RPMI-1640 medium (HyClone, Logan, UT, USA) with 10\% FBS (HyClone, Logan, UT, USA) and cultured in an incubator maintained with $5 \% \mathrm{CO}_{2}$ at $37^{\circ} \mathrm{C}$.

\section{Immunohistochemistry}

FFPE specimens were cut into $4-5-\mu \mathrm{m}$ sections. The immunohistochemistry (IHC) procedure was performed as described previously [13]. Briefly, the sections were incubated with a rabbit anti-SYT7 polyclonal antibody (1:200 dilution; Abcam, Cambridge, UK) overnight at $4^{\circ} \mathrm{C}$. After washing with phosphate-buffered saline (PBS), biotinylated goat anti-mouse secondary antibody was applied, and slides were incubated for $30 \mathrm{~min}$ at room temperature. SYT7 expression was visualized with 3,3'-diaminobenzidine(DAB) (MaiXin, Fuzhou, China). Tissue sections were examined under a microscope, and evaluated for both staining intensity and percentage of positive cells. Staining intensity was classified as follows: 1 (weak), 2 (moderate), or 3 (strong). The percentage of positive cells was scored as $0(\leq 5 \%), 1$ (6-25\%), $2(26-50 \%), 3(51-75 \%)$, and $4(76-100 \%)$. The staining intensity and percentage of stained cells were then multiplied to generate the immunoreactivity score for each case, ranging from 0 to 12 . Tumor tissues with an immunoreactivity score of $\geq 4$ were considered to have high expression, and those with a score of $<4$ were considered to have low expression.

\section{RNA interference and construction of stable cell line}

Small interfering RNA (shRNA)(target sequence: 5'-TCACCGTGAAGATCATGAA-3') targeting human SYT7 was designed and the scrambled shRNA(target sequence: 5'-TTCTCCGAACGTGTCACGT-3') was used as negative control. Lentivirus expressing such SYT7 shRNA and scrambled shRNA were constructed (GeneChem, Shanghai, China). Transfection was performed in RKO cells using Lipofectamine 2000 (Invitrogen, Grand Island, NY) as the manufacturer's protocol. After 72 hours, the transfection efficiency could be detected using fluorescence microscope. Then cells were harvested and total RNA and protein were extracted to determine the knockdown efficiency using real-time PCR and western blot. Finally, LV transfected RKO cells were quickly collected for later experiment.

\section{Quantitative real-time PCR}

Total RNA was isolated by Trizol reagent (Takara Bio, Otsu, Japan). cDNA was synthesized from total RNA using the Expand Reverse Transcriptase Kit (Thermo Fisher Scientific, Waltham, MA, USA). The amplification was performed under the following conditions: $95^{\circ} \mathrm{C}$ for $15 \mathrm{~s}, 45$ cycles of $95^{\circ} \mathrm{C}$ for $5 \mathrm{~s}$ and $60^{\circ} \mathrm{C}$ for $30 \mathrm{~s}$. Diethyl pyrocarbonate (DEPC) water was used in place of the template cDNA for the negative control. The primers were as follows: SYT7, 5'- ACTCCATCATCGTGAACATCA TC -3' (sense) and 5' - TCGAAGGCGAAGGACTCAT TG-3' (antisense); GADPH, 5'-TGACTTCAACAGC GACACCCA-3' (sense) and 5'-CACCCTGTTGCTG 
TAGCCAAA-3' (antisense). The expression level was determined using the $2^{-\Delta \Delta \mathrm{Ct}}$ method (relative quantification). Each experiment was performed in triplicate.

\section{Western blot analysis}

Whole protein lysates were prepared from cell line using RIPA lysis buffer (Solarbio, Beijing, China). Samples were loaded into 10\% SDS-PAGE gels, transferred to polyvinylidene difluoride(PVDF) membranes (Millipore Corp, Bedford, MA, USA) and incubated with primary anti-SYT7 (1:500 dilution) and anti-GAPDH(1:2000 dilution; Santa Cruz, CA, USA) overnight at $4^{\circ} \mathrm{C}$. Membranes were incubated with secondary monoclonal antibody (1:2000 dilution; Santa Cruz, CA, USA) at room temperature for 2 hours. Protein bands were visualized using an ECL detection kit (ECL, Thermol Biotech Inc, USA).

\section{Cell proliferation analysis using Celigo image cytometer}

RKO cells were collected after infected with SYT7-shRNA or Scr-shRNA for $72 \mathrm{~h}$. Then, cells were plated into 96-well plates at a density of 1,000 cells/well in triplicate. After culturing in a $5 \% \mathrm{CO}_{2}$ incubator at $37^{\circ} \mathrm{C}$ for another one day, cell images were captured using Celigo image cytometer (Nexcelom Bioscience, Lawrence, MA, USA) once a day for 5 days. Cell numbers in each well were also quantified by using Celigo image cytometer and cell growth curves were created for each group.

\section{Clonogenic formation assay}

RKO cells were collected after infected with SYT7-shRNA or Scr-shRNA for $72 \mathrm{~h}$. Cells were plated into six-well plates at a density of 500 cells/well in triplicate. After incubation in a $5 \% \mathrm{CO} 2$ incubator at $37^{\circ} \mathrm{C}$ for 11 days, cells were fixed with 1 $\mathrm{ml} 4 \%$ paraformaldehyde for $45 \mathrm{~min}$. Cell colony was then stained with Giemsa(AR-0752, Shanghai) for 15 min and washed with DdH2O. Images of cell colony were captured by Fluorescence microscope (IX71, Olympus, Tokyo, Japan).

\section{Cell Cycle Analysis}

After achieving nearly $70 \%$ confluent, cells infected with SYT7-shRNA or Scr-shRNA were harvested and washed using pre-cold HBSS (PH: 7.2-7.4; Sigma, St. Louis, MO, USA) and then fixed with ice-cold $75 \%$ alcohol for at least $1 \mathrm{~h}$. Cells were then re-suspended in $1000 \mu \mathrm{L}$ HBSS containing 50 $\mu \mathrm{g} / \mathrm{ml}$ of Propidium Iodide (Sigma, St. Louis, MO, USA) and $100 \mu \mathrm{g} / \mathrm{ml}$ of RNase. Flow cytometry was performed using FACS Calibur (Guava easyCyte HT, Millipore,USA) for cell cycle analysis. Each experiment was performed in triplicate.

\section{Apoptosis Assay}

Cell apoptosis was detected by using Annexin V-APC apoptosis detection kit (eBioscience, San Diego, CA, USA) following the manufacturer's instructions. RKO cells were treated as above. After incubation for another 5 days, cells were harvested, and resuspended using $1 \times$ Binding buffer at a final density of $1-2 \times 10^{6} / \mathrm{ml}$. Then $10 \mu \mathrm{l}$ annexin V-APC was added into $200 \mu \mathrm{l}$ cell suspensions and incubated at room temperature protected from light for 10-15 min. Signals were detected with FACS Calibur.

\section{Microarray and Ingenuity Pathway Analysis}

The quality of total RNA samples was evaluated with an Agilent Bioanalyzer 2100 (Agilent, Santa Clara, CA, USA) and the purified RNA was quantified using a NanoDrop 2000(Thremo Fisher Scientific, Waltham, MA, USA). And human GeneChip primeview (Affymetrix, Santa Clara, CA, USA) was used for microarray processing to determine gene expression according to the manufacturer's instructions. Significant differentially expressed genes between RKO cells infected with SYT7-shRNAs and Scr-shRNAs were chose according to the following principles: $\mathrm{P}$ value $<0.05$ and absolute fold change (FCAbsolute) $>1.5$. Ingenuity Pathway Analysis (IPA) was executed for all significant differentially expressed genes that were based on pathway databases [14]. $\mid Z$ - score $\mid>2$ is considered to be significant. The positive $\mathrm{Z}$ - score means that this pathway is significantly activated. While the negative $\mathrm{Z}$ - score means that this pathway is significantly suppressed [15].

\section{Statistical analysis}

Data are presented as means \pm S.D. and statistical analysis was performed using Student's $\mathrm{t}$ test or one-way ANOVA (SPSS Statistics, version 19.0, Chicago). $\quad \mathrm{P}<0.05$ were considered statistically significant. The data graphs were made with GraphPad Prism 6.0 software.

\section{Results}

\section{Bioinformatics analysis suggests SYT7 as a potential oncogene in colorectal cancer}

Among the TCGA-colorectal adenocarcinoma datasets, genes with a fold-change $>2$ were involved in our analysis. By searching the literature, we finally identified SYT7 as a candidate gene. SYT7 expression was 3.01 fold hyper-expressed in cancer tissues $(\mathrm{P}=1.82 \mathrm{E}-20)$ (Figure 1A, B) than normal colorectal tissues. Although SYT7 was noted as a calciumdependent regulator in synaptic transmission in the NCBI database, the function of SYT7 in cancer progression is unclear. 
A

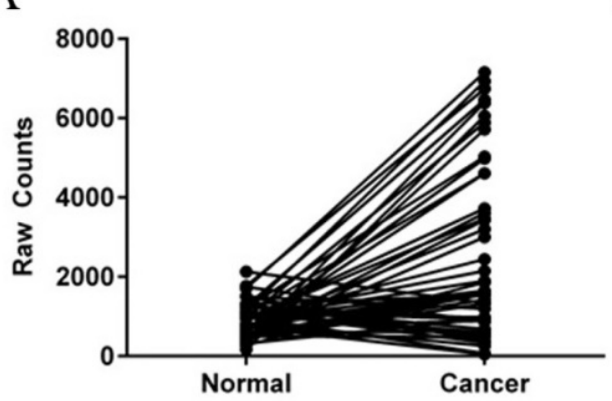

B

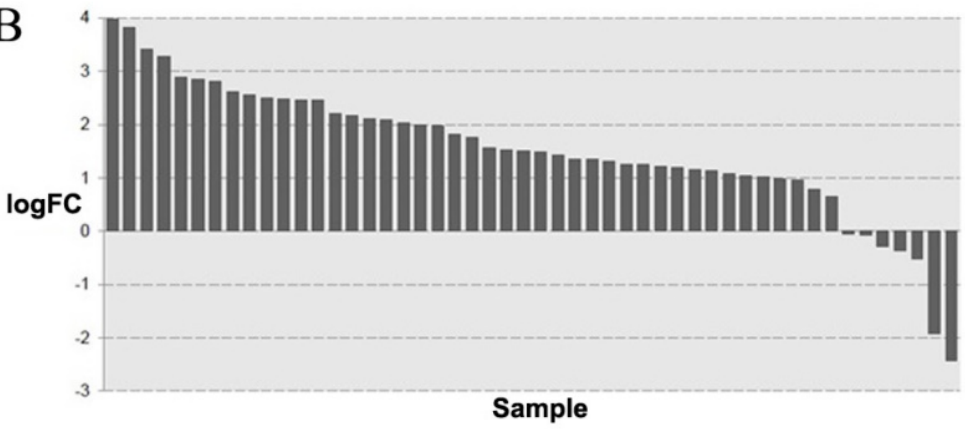

Figure 1. SYT7 is highly expressed in colorectal cancer tissues. (A) After analysis of 50 paired tissues in TCGA database, SYT7 was found to be highly expressed in tumors. (B) SYT7 is over-expressed in $78 \%$ (39 of 50$)$ of the colorectal cancer tissues, with an average over-expression of 3.01 -fold (P<0.001) as compared to paired normal tissues.

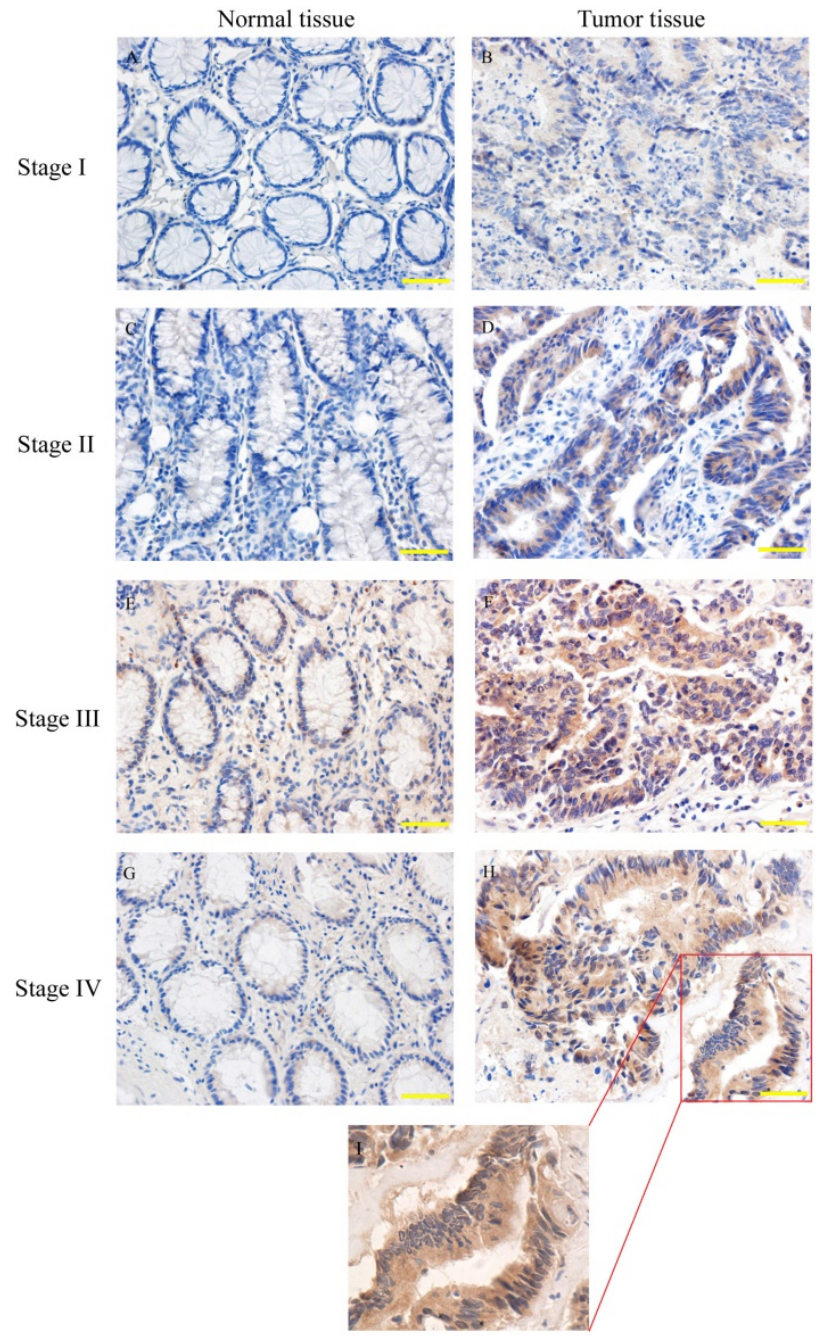

Figure 2. SYT7 expression in colorectal cancer of different stages. SYT7 expression was detected by immunohistochemical staining(IHC) A,C,E,G: normal tissues; B,D,F,H: tumor tissues from stage I to stage IV (magnification, $\times 200$ ); I: SYT7 expression in colorectal cancer, (magnification, $\times 400$ )

\section{SYT7 is overexpressed in colorectal cancer}

Human colorectal cancers have been classified into I to IV stage according to clinical-pathological parameters. SYT7 expression was analyzed in 83 pairs of tumor and normal tissues by using IHC (Fig. 2).
The results showed that SYT7 expression was rarely detected in normal tissues, while the positive expression rate was $50.6 \%(42 / 83)$ in tumor tissues. And the rate was higher in colorectal cancer with higher stage (Table 1). These results showed a significant positive correlation between SYT7 expression and stage of colorectal cancer $(\mathrm{P}=0.015)$.

Table 1. The clinical and pathological characteristics of the patients

\begin{tabular}{|c|c|c|c|c|c|c|}
\hline & \multirow[t]{2}{*}{ variables } & \multicolumn{2}{|c|}{ SYT7 expression } & \multirow[t]{2}{*}{ total } & \multirow[t]{2}{*}{ rs } & \multirow[t]{2}{*}{$\mathrm{p}$ value } \\
\hline & & low & high & & & \\
\hline \multirow[t]{3}{*}{ Sex } & & & & & 0.037 & 0.827 \\
\hline & Male & 23 & 22 & 45 & & \\
\hline & Female & 18 & 20 & 38 & & \\
\hline \multirow[t]{3}{*}{ Age(year-old) } & & & & & -0.035 & 0.827 \\
\hline & $\leq 60$ & 22 & 24 & 46 & & \\
\hline & $>60$ & 19 & 18 & 37 & & \\
\hline \multirow[t]{3}{*}{ Size $(\mathrm{cm})$} & & & & 0.076 & & 0.615 \\
\hline & $<5$ & 32 & 30 & 62 & & \\
\hline & $\geq 5$ & 9 & 12 & 21 & & \\
\hline \multirow[t]{4}{*}{ Grade } & & & & & 0.108 & 0.333 \\
\hline & 1 & 10 & 2 & 12 & & \\
\hline & 2 & 25 & 38 & 63 & & \\
\hline & 3 & 6 & 2 & 8 & & \\
\hline \multirow[t]{3}{*}{ T stage } & & & & & 0.034 & 0.810 \\
\hline & $\mathrm{T} 1+\mathrm{T} 2$ & 12 & 11 & 23 & & \\
\hline & $\mathrm{T} 3+\mathrm{T} 4$ & 29 & 31 & 60 & & \\
\hline \multirow[t]{3}{*}{ N stage } & & & & & 0.261 & $0.025^{*}$ \\
\hline & No & 30 & 20 & 50 & & \\
\hline & N1 & 11 & 22 & 33 & & \\
\hline \multirow[t]{3}{*}{ M stage } & & & & & 0.144 & 0.313 \\
\hline & M0 & 38 & 35 & 73 & & \\
\hline & M1 & 3 & 7 & 10 & & \\
\hline \multirow[t]{3}{*}{ UICC stage } & & & & & 0.281 & $0.015^{*}$ \\
\hline & I-II & 29 & 18 & 47 & & \\
\hline & III-IV & 12 & 24 & 36 & & \\
\hline
\end{tabular}

* Statistically significant $(\mathrm{p}<0.05)$

\section{Knockdown of SYT7 inhabits RKO cells proliferation and colony formation in vitro}

To evaluate the role of SYT7, we knocked down SYT7 in RKO cells by using Lentiviral-shRNA. 72 hours after transfection, the rate of infected cell in both two groups was about $80 \%$. And then we detected the SYT7 expression by using qt-PCR and 
western blot. SYT7 expression was obviously inhibited at protein level in RKO cells treated with SYT7-shRNA (Fig. 3A) and was reduced by about 65.4 $\%$ at mRNA level compared to the untreated cells (P=1.1E-5)(Fig. 3B). These results revealed that SYT7 expression was efficiently inhibited by LentiviralshRNA in RKO cells. Celigo image cytometer was used to record the daily proliferation of cells for 5 days. Cells treated with SYT7-shRNA showed significant growth inhibition from the third day compared to the control group (Day 5: $4979 \pm 448$ vs $796 \pm 29, \mathrm{P}=8.6 \mathrm{E}-5)$. In addition, the inhibition effect of SYT7-shRNA on cell proliferation was more pronounced as the incubation time increased (Fig. 3C). Furthermore, we studied the function of SYT7 knockdown on RKO cells tumorigenesis in vitro by assaying colony formation. Results showed that SYT7

knockdown significantly suppressed cell colony formation. In Brief, there were about 184 clones formed in cells treated with Scr-shRNA compared to 37 treated with SYT7-shRNA on the average (Fig. 3D). The reduction rate of clonogenic ability of RKO cells treated with SYT7-shRNA was about $79.9 \%$ (184 \pm 6 vs $37 \pm 5, \mathrm{P}=4.5 \mathrm{E}-6)$.

\section{SYT7 knockdown promots G2/M Phase arrest and apoptosis in RKO cells}

As shown in (Fig. 4A), cell percentage at G2/M phase was increased from $9.82 \%$ in control group to $22.84 \%$ in SYT7-shRNA treated cells $(9.82 \pm 0.20$ vs $22.84 \pm 0.88, \mathrm{P}=1.5 \mathrm{E}-5)$, while the cell percentage at $\mathrm{S}$ phase was decreased from $38.95 \%$ in control group to $28.63 \%$ in SYT7-shRNA treated cells $(38.95 \pm 1.13$ vs $28.63 \pm 0.72, \mathrm{P}=0.00018)$. So these results indicated that

A
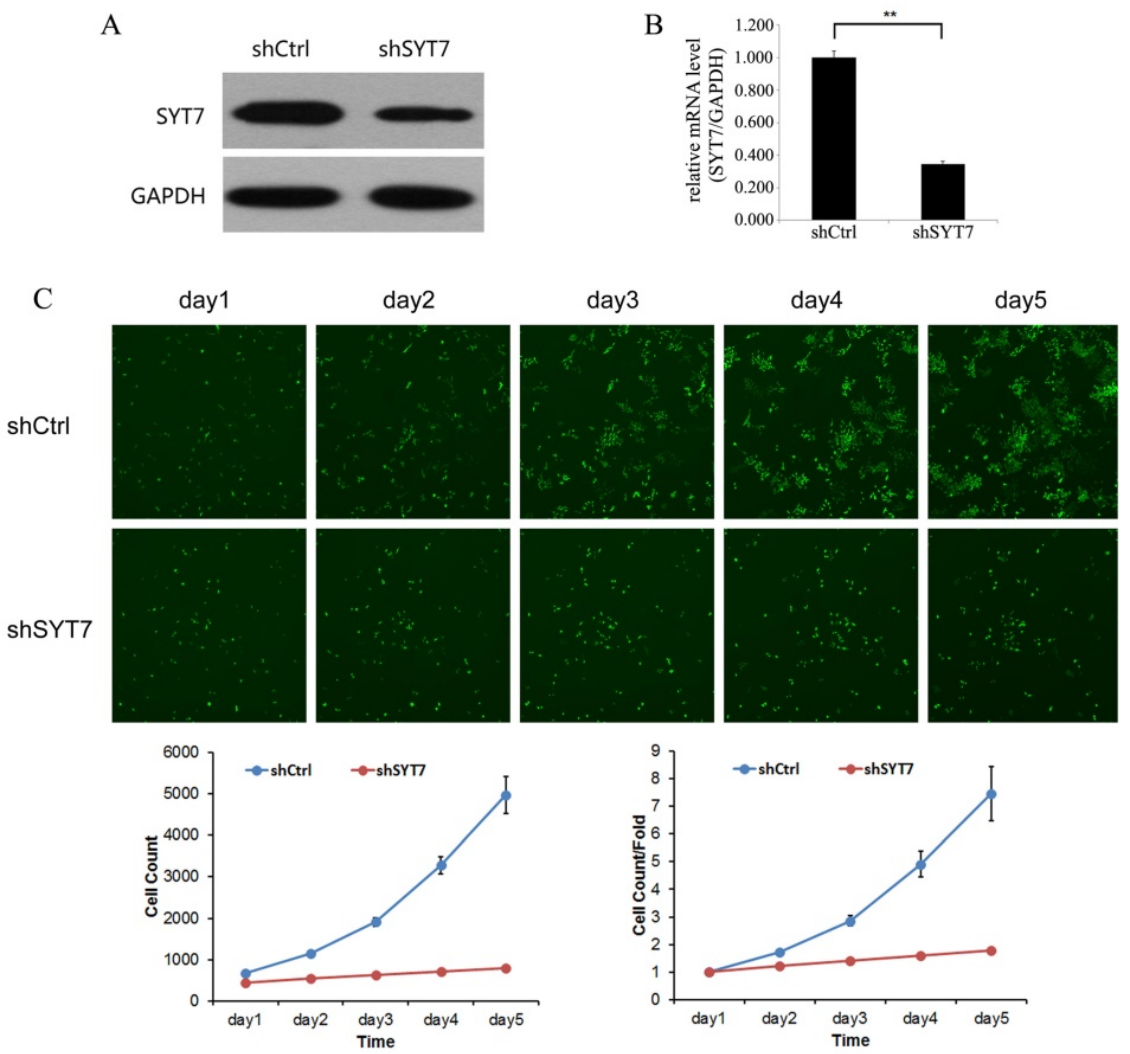

D
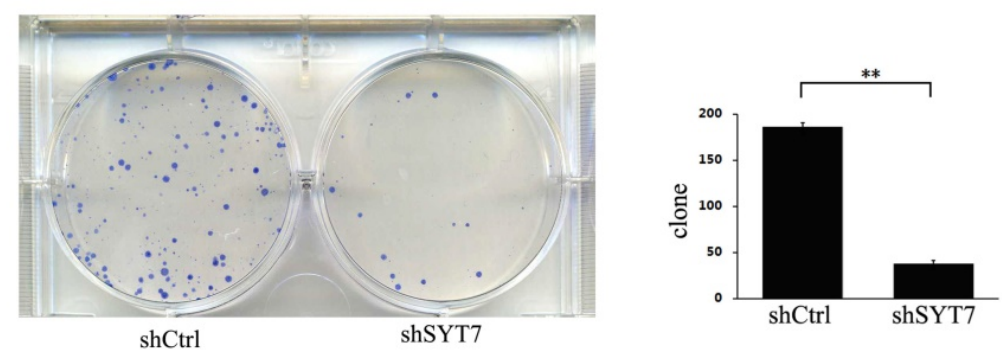

Figure 3. Knockdown of SYT7 inhabited RKO cells proliferation and colony formation in vitro. (A) SYT7 expression change at protein level was analyzed by western blot in RKO cells treated as described after 4 days. GAPDH was used as an internal. (B) SYT7 expression change at mRNA level was detected by real-time quantitative PCR. SYT7 mRNA level was normalized to GAPDH. Data shown are the mean $\mathrm{S} . \mathrm{D}$. of three independent experiments. **: P<0.01. (C) Impaired cell proliferation after SYT7 knockdown in RKO cells were detected by Celigo image cytometer. **: $P<0.01$. (D) Clonogenic ability was impaired in RKO cells by SYT7 knockdown using colony formation assay. The results represent mean \pm S.D. of three separate experiments. $* *$ : P<0.01. 

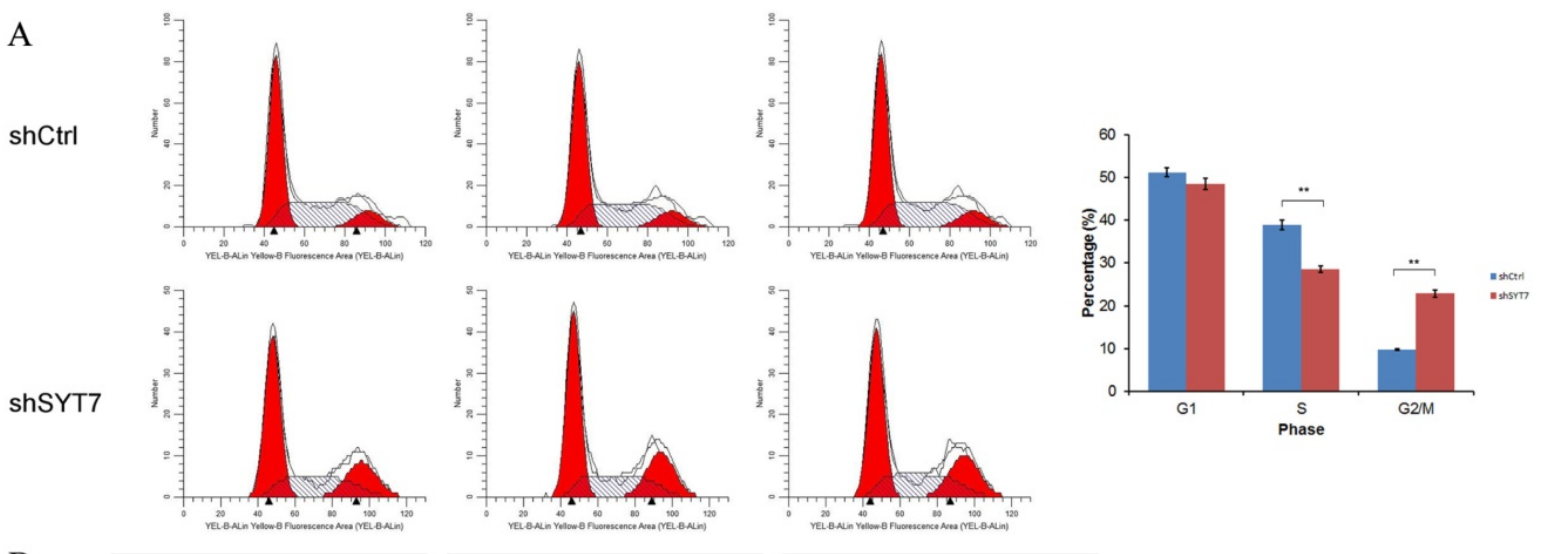

B

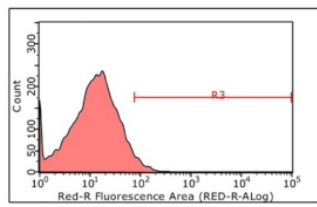

ANNEVIN-V

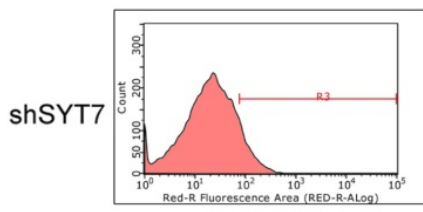

ANNEVIN-V

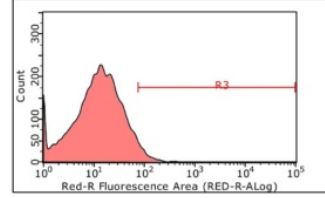

ANNEVIN-V

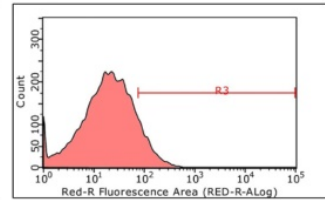

ANNEVIN-V

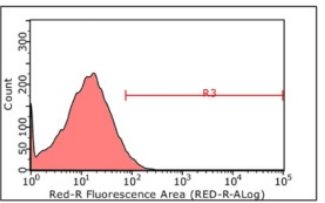

ANNEVIN-V

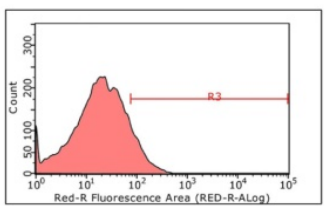

ANNEVIN-V

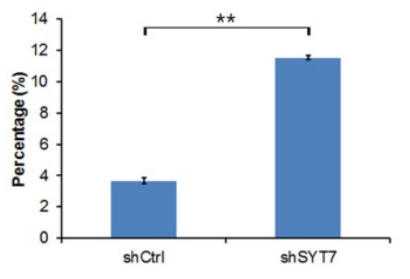

Figure 4. Cell cycle arrest and apoptosis were induced in RKO cells by SYT7 knockdown. (A) The changes in the cell cycles of RKO cells by SYT7 knockdown, the percentage of cells in the $S$ phase significantly increased. Graph shows the mean $\pm S$.D. of cell proportion in the G1 phase, $S$ phase and G2/M phase from three separate experiments. **: $\mathrm{P}<0.01$ in $\mathrm{S}$ phase and $\mathrm{G} 2 / \mathrm{M}$ phase. (B) The changes in the apoptosis rates of RKO cells by SYT7 knockdown. Data shown here is the mean \pm S.D. of cell percentage in apoptosis from three separate experiments. $* *: P<0.01$.

SYT7 plays an important role in G2-M transition in RKO cells. The percentage of apoptotic cells was significantly higher in treated with SYT7-shRNA group (Fig. 4B). The apoptosis rate of RKO cells was increased from $3.65 \%$ to $11.53 \%$ after infected with

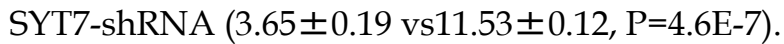

\section{Genes expression in multiple cancer-associated pathways changed after SYT7 knockdown}

1915 genes showed significant differential expressions after RKO cells treated with SYT7-shRNA (FCAbsolute>1.5 and $\mathrm{P}<0.05$ ), including 978 unregulated and 937 downregulated (Fig. 5A). Ingenuity pathway analysis was executed and found that genes expression in these fourteen signaling pathways change significantly. Many of these pathways played an important role in cancer development and progression (Fig. 5B). Two suppressed signaling pathways, ATM signaling and mitotic roles of polo-like kinase, significantly changed after SYT7 knockdown respectively. Functional interaction network analysis was applied to inquire into the relation between genes that belong to these two pathways (Fig. 5C, D). G2/M DNA Damage Checkpoint Regulation pathway was activated by
SYT7 knockdown. Majority of SYT7-regulated genes expression in ATM signaling (down/up ratio=15/4), mitotic roles of polo-like kinase (down/up ratio $=17 / 1$ ) and G2/M DNA Damage Checkpoint Regulation (down/up ratio=13/2) pathways were downregulated by SYT7 knockdown. Among these genes, CDK1 (FCAbsolute $=1.80, \mathrm{P}=0.00011)$, PLK1 (FCAbsolute $=2.16, \quad \mathrm{P}=0.00017$ ) and cyclin $\mathrm{B}$ (FCAbsolute=1.73, $\mathrm{P}=0.00008$ ) respectively in these three signal pathways were well-known for their oncogenic effect on cancer development.

\section{Discussion}

Currently, surgical resection, chemotherapy, radiotherapy, and other curative strategies are applied to cure colorectal cancer. In spite of the advances in screening, diagnosis, and treatment, some patients with colorectal cancer have poor prognosis due to lymph node metastasis (LNM) and distant metastasis [16]. Therefore, it is of great significance to further investigate the molecular mechanism of occurrence, development, and metastasis in colorectal cancer. Molecular genetics studies have revealed some critical tumor-associated genes underlying the progression of colorectal cancer [17]. 
A

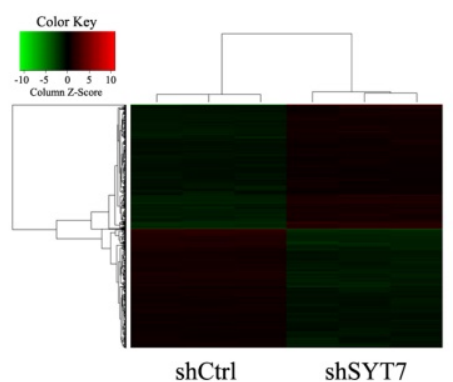

C

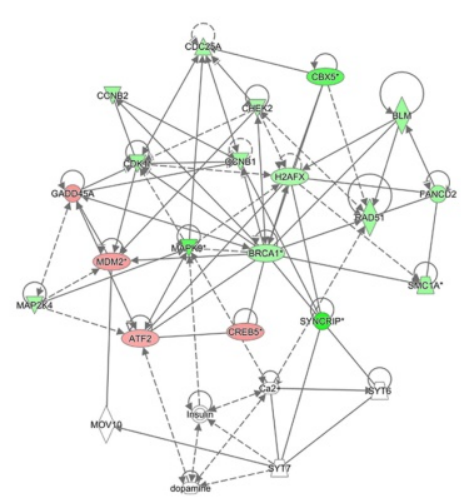

B

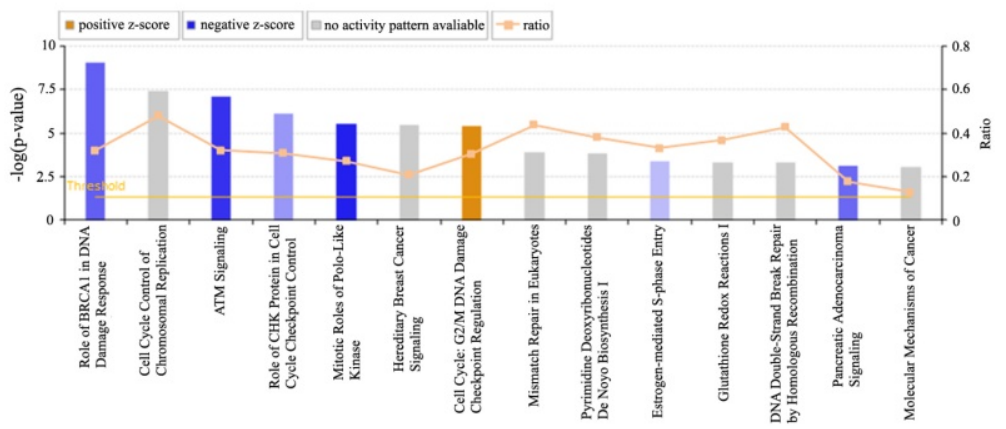

D

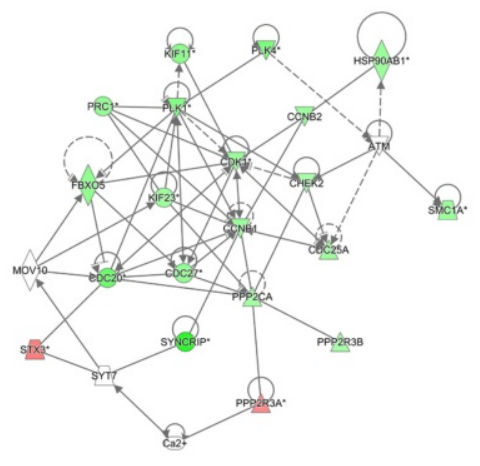

Figure 5. Widespread changes of gene expressions in RKO cells with SYT7 knockdown. (A) Representation of 1915 genes showed significant differential expressions in RKO cells infected with lentivirus expressing either Scr-shRNA or SYT7-shRNA. A color scale for the normalized expression data was shown at the upper left corner of the microarray heatmap (green represents downregulated genes while red represents upregulated genes). (B) The signal pathway histogram shows the enrichment of the differential gene in the classical signaling pathway based on IPA pathway. All signal paths were sorted using -Log ( $\mathrm{P}$-value). Here, the 14 significant-enriched pathways based on a $\mathrm{P}<0.05$ were shown. Z-score $>2$ represents that the Pathway is significantly activated, while Z-score <-2 represents that the Pathway is significantly inhibited. Ratio represents the ratio of differentially expressed genes numbers to all gene numbers in this signal pathway. Networks were constructed between SYT7 and genes involved in ATM signaling (C) and mitotic roles of polo-like kinase (D), respectively. Green color represents downregulated genes and red color represents unregulated genes. Solid arrow in the figure means confirmed regulatory relationships and dotted line means predicted regulatory relationships. Inhibitory relationship was represented by "T" bar.

SYT7 belongs to the synaptotagmin gene family and encodes a protein like other family members mediating calcium-dependent regulation of membrane that traffics in synaptic transmission [18]. Shih et al. [19] reported that the functional SYT7 is critical for the regulation of norepinephrine releasing from cardiac sympathetic nerve endings. Peng et al. [20] studied PLXNC1's role in collagen accumulation and macrophage migration. They found that PLXNC1 deficiency permited SYT7-mediated macrophage migration and enhanced mammalian lung fibrosis. Both of these roles could be improved by SYT7's genetic deletion. This discovery demonstrated that SYT7 plays an important role in mediating macrophage migration. However, the expression pattern and cellular localization of SYT7 protein, its clinical significance, and its mechanism in the progression of colorectal cancer remain poorly understood. In this study, we found that SYT7 expression was upregulated in colorectal cancer tissues and the overexpression rate of SYT7 was positively correlated with colorectal cancer stages. These results suggested that SYT7 may participate in the progression of colorectal cancer.
To assess whether SYT7 expression in CRC tissues served as a therapeutic marker for CRC, we made an optimal knockdown of SYT7 in RKO cells. Using Celigo image cytometer, clonogenic formation assay, apoptosis and cell cycle assay, we found that SYT7 knockdown suppresses tumorigenesis and cell proliferation, and promotes apoptosis and G2/M phase cell cycle arrest. In order to further explore the roles of SYT7-mediated development and progression of colorectal cancer, we made the microarray analysis for RKO cells with SYT7 knockdown and found that many genes had significant changes. We next examined the gene functions by using functional pathway analysis and found that many signal pathways changed after SYT7 knockdown. For example, PLK1-CHEK2 pathway, the top-inhibited pathway after SYT7 knockdown, is very important for cancer cell proliferation and migration [21]. ATM signaling pathway, which is critical for DNA damage response, has participated in the development of many tumors and possible targets for cancer treatment [22]. In addition, many well-known oncogenes such as CDK1 and PLK1 were remarkable downregulated after SYT7 knockdown. These results provide a potential evidence of the important role of 
SYT7 in the development of colorectal cancer and suggest that SYT7 may be an upstream factor in the development and progression of cancer. The G2-M DNA damage checkpoint is an important cell cycle checkpoint in eukaryotic organisms. This checkpoint ensures that cells don't initiate mitosis before they have an opportunity to repair damaged DNA after replication [23]. The activation of G2/M DNA Damage Checkpoint Regulation Pathway may explain the reason of G2/M phase arrest by SYT7 knockdown. Future study in detailed molecular mechanisms will well unravel the role of SYT7 in tumor development.

In Conclusion, this is the first research that links SYT7 to colorectal cancer progression. Our study suggests that SYT7 is overexpressed in colorectal cancer and regulates colorectal cancer cell proliferation. Further detailed investigations into SYT7 functions based on the microarray analysis results will deepen the understanding about causal roles of SYT7 in colorectal cancer progression and provide a potential therapeutic target.

\section{Competing Interests}

The authors have declared that no competing interest exists.

\section{References}

1. Brenner H, Kloor M, Pox CP. Colorectal cancer. The Lancet. 2014; 383: 1490-502.

2. Chen $W$, Zheng R, Baade PD, Zhang S, Zeng H, Bray F, et al. Cancer statistics in China, 2015. CA Cancer J Clin. 2016; 66:115-32.

3. Chen J, Zhang D, Zhang W, Tang Y, Yan W, Guo L, et al. Clear cell renal cell carcinoma associated microRNA expression signatures identified by an integrated bioinformatics analysis. J Transl Med. 2013; 11:169.

4. Prensner JR, Iyer MK, Balbin OA, Dhanasekaran SM, Cao Q, Brenner JC, et al. Transcriptome sequencing across a prostate cancer cohort identifies PCAT-1, an unannotated lincRNA implicated in disease progression. Nat Biotechnol. 2011; 29:742-49.

5. Wang X, Sun Q, Chen C, Yin R, Huang X, Wang X, et al. ZYG11A serves as an oncogene in non-small cell lung cancer and influences CCNE1 expression. Oncotarget. 2016; 7:8029-42.

6. Chapman ER. How does synaptotagmin trigger neurotransmitter release? Annu Rev Biochem. 2008; 77:615-41.

7. Segovia M, Alés E, Montes MA, Bonifas I, Jemal I, Lindau M, et al. Push-and-pull regulation of the fusion pore by synaptotagmin-7. Proc Natl Acad Sci USA. 2010; 107:19032-7.

8. Gustavsson N, Wei SH, Hoang DN, Lao Y, Zhang Q, Radda GK, et al. Synaptotagmin-7 is a principal $\mathrm{Ca} 2+$ sensor for $\mathrm{Ca} 2+$-induced glucagon exocytosis in pancreas. J Physiol. 2009; 587:1169-78

9. Weber JP, Toft-Bertelsen TL, Mohrmann R, Delgado-Martinez I, Sørensen JB. Synaptotagmin-7 is an asynchronous calcium sensor for synaptic transmission in neurons expressing SNAP-23. PLoS One. 2014; 9:e114033.

10. Neuland K, Sharma N, Frick M. Synaptotagmin-7 links fusion-activated $\mathrm{Ca}^{2+}$ entry and fusion pore dilation. J Cell Sci. 2014; 127:5218-27.

11. Liu H, Bai H, Hui E, Yang L, Evans CS, Wang Z, et al.. Synaptotagmin 7 functions as Ca2+-sensor for synaptic vesicle replenishment. Elife. 2014; 3:e01524.

12. Sobin LH, Gospodarowicz MK, Wittekind C. TNM classification of malignant tumors, 7th ed. Wiley-Blackwell, Oxford. 2010.

13. Wang K, Dong M, Sheng W, Liu Q, Yu D, Dong Q, et al. Expression of vitamin $\mathrm{D}$ receptor as a potential prognostic factor and therapeutic target in pancreatic cancer. Histopathology. 2015; 67:386-97.

14. Krämer A, Green J, Pollard J, Tugendreich S. Causal analysis approaches in Ingenuity Pathway Analysis. Bioinformatics. 2014; 30:523-30.

15. Felciano RM, Bavari S, Richards DR, Billaud JN, Warren T, Panchal R, et al. Predictive systems biology approach to broad-spectrum, host-directed drug target discovery in infectious diseases. Pac Symp Biocomput. 2013; pp: 17-28.

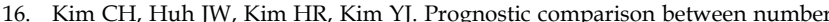
and distribution of lymph node metastases in patients with right-sided colon cancer. Annals of Surgical Oncology. 2014; 21: 1361-8.
17. Fearon ER. Molecular genetics of colorectal cancer. Annu Rev Pathol. 2011; 6: 479-507.

18. Jackman SL, Turecek J, Belinsky JE, Regehr WG. The calcium sensor synaptotagmin 7 is required for synaptic facilitation. Nature. 2016; 529:88-91.

19. Shih AM, Varghese L, Bittar A, Park SH, Chung JM, Shin OH. Dysregulation of norepinephrine release in the absence of functional synaptotagmin 7. J Cell Biochem. 2016; 117:1446-53.

20. Peng X, Moore M, Mathur A, Zhou Y, Sun H, Gan Y, et al. Plexin C1 deficiency permits synaptotagmin 7 -mediated macrophage migration and enhances mammalian lung fibrosis. FASEB J. 2016; 30:4056-70.

21. Han DP, Zhu OL, Cui JT, Wang PX, Qu S, Cao OF, et al. Polo-like kinase 1 is overexpressed in colorectal cancer and participates in the migration and invasion of colorectal cancer cells. Med Sci Monit. 2012; 18:237-46.

22. Weber AM, Ryan AJ. ATM and ATR as therapeutic targets in cancer. Pharmacol Ther. 2015; 149:124-38.

23. Cuddihy AR, O'Connell MJ. Cell-cycle responses to DNA damage in G2. Int Rev Cytol. 2003; 222:99-140. 